\title{
Fresh Properties of mercapto Chitosan in Cherry preservation
}

\author{
Li Yu-feng \\ Key Laboratory of Fine Flake Graphite Deep Processing, Panzhihua College, \\ Panzhihua ,Sichuan Province, PR China \\ lyfpzh@163.com
}

\section{Key words: Cherry; Chitosan; preservation}

Abstract: To obtain a good Cherry preservative, the author prepared mercapto Chitosan and metal ion complexes with solid-liquid reaction. Besides, the Cherry preservative was prepared by modified Chitosan materials. It was obtained that the optimal concentration of chitosen acetate for fresh cherries is $10 \mathrm{~g} / \mathrm{l}$ by studying effect of different concentrations of preservative on the storage of Cherry and its preservation time was 21 days. It also found that it owned better preservation effect with adding metal ion and $\mathrm{Zn}$ compound preservative had the best preservation performance among all experimental ion compound preservation. The experiment results showed that the preservative which prepared by Chitosan had excellence properties of extending Cherries storage periods、 lowing the loss of Cherry fruit during storage、slowing the forming of Cherry soluble solids and the decreasing of titratable acid and vitamine $\mathrm{C}$, but also reducing Cherry metabolism.

\section{Introduction}

Cherry fruit ripe earlier among deciduous fruit trees and is named "mince first". Cherry fruit plays important role in filling the supply gap, enriching and balancing fruit anniversary supply to meet demand for human consumption in fresh fruit market in the late spring and early summer every year. Cherry fruit are praised as "rare fruit" for its delicious color and abundant nutrition. Cherry fruit can promote the regeneration of hemoglobin and it is good for patient with anemia v corneal disease and dry skin. However, it is difficult in storage and transportation because of its poor anti-extrusion capabilities and natural preservation. Therefore, studying ways of Cherry preservation attracts more and more working of scientists. It investigates the preservation effect of mercapto Chitosan and its metal ion complex via the indicators of loss ratev soluble solids and titratable acid content.

\section{Experiment}

Materials. Chitosan(GMP Shandong, China), Sodium hydroxide(AR Chengdu, China), Potassium permanganate( AR Chengdu, China), Mercapto acid(AR Chengdu, China)

Apparatus. Magnetic stirrer (SZCL-A, Henan, China), Analytical balance (FA2004, Shanghai, China), Centrifuge (TDL-40B, Shanghai, China)

\section{Preparation of mercapto Chitosan}

Chitosan powder $(20 \mathrm{~g})$ dispersed in $100 \mathrm{~mL}$ ethanol solution $(95 \%)$ for 8 hours, then filtered to remove ethanol aqueous solution, slowly adding $20 \mathrm{~mL}$ mercapto acid (AR) and continue stirring $3 \mathrm{~h}$ at $25{ }^{\circ} \mathrm{C}$, and then washed by ethanol, dried in a vacuum oven to constant weight and obtaining dark yellow mercapto Chitosan product. 


\section{Preparation of modified Chitosan metal ion complexes}

Weighing a certain amount of modified Chitosan material in $100 \mathrm{ml}$ conical flask and adding $20 \mathrm{ml}$ solvent with cylinder volume, then shaking around 30min, quantitative solid calcium chloride powder is dropped in conical flask and is shaken to make mixed units dissolve. A high boiling point liquid placed in microwave oven to maintained temperature at $90-100^{\circ} \mathrm{C}$ and put the conical flask in to heat for a certain time. After that, the conical flask is taken out and quick cooling. Then, slowly adding some ascorbic acid vglucose during stirring and adjusting the $\mathrm{pH}$ to 3-4 with acetic acid to obtain fruit preservation. The preparation of other metal ions complexes are the same as above.

\section{Results and discussion}

Cherry sample were coated by mercapto chitosan solution and its metal ion complexes and dried in the air to form surface membrane.

\section{The coating effect of mercapto chitosan and its metal complexes on loss rate of cherry}

The figure 1 shows a obvious difference of the loss rate of cherry during days before and there is a little difference between different sample. They all reduced the loss of the cherry.

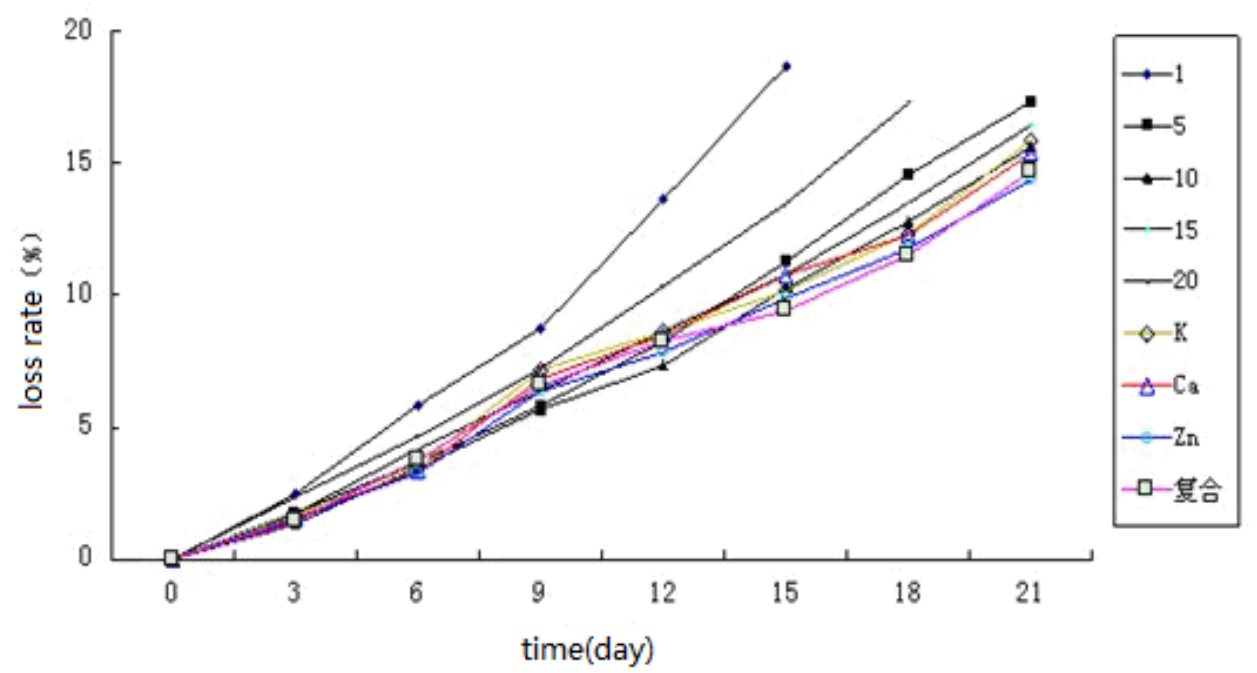

Fig 1 Effect on loss rate of cherry

The coating effect of mercapto chitosan and its metal complexes on soluble solids content of cherry

The figure 2 shows the effect of CS film on cherry soluble solids content. The soluble solids content of control group decrease significantly from beginning, while that of CS-coated film increase firstly and decrease slowly. The reason of this phenomenon is that preservation with adding glucose makes cherry sugar levels rise rapidly in the beginning. The experimental group with single film has the best effect at the concentration of $10 \mathrm{~g} / \mathrm{l}$ and its soluble solids content is $15.3 \%$ after 21 days storage, even higher than fresh cherries. In the use of potassium vinc and calcium metal ion complexes in the experimental group, the soluble solids contents is more than $15.7 \%$ after 21 days storage, are higher than that of single CS coating group. 


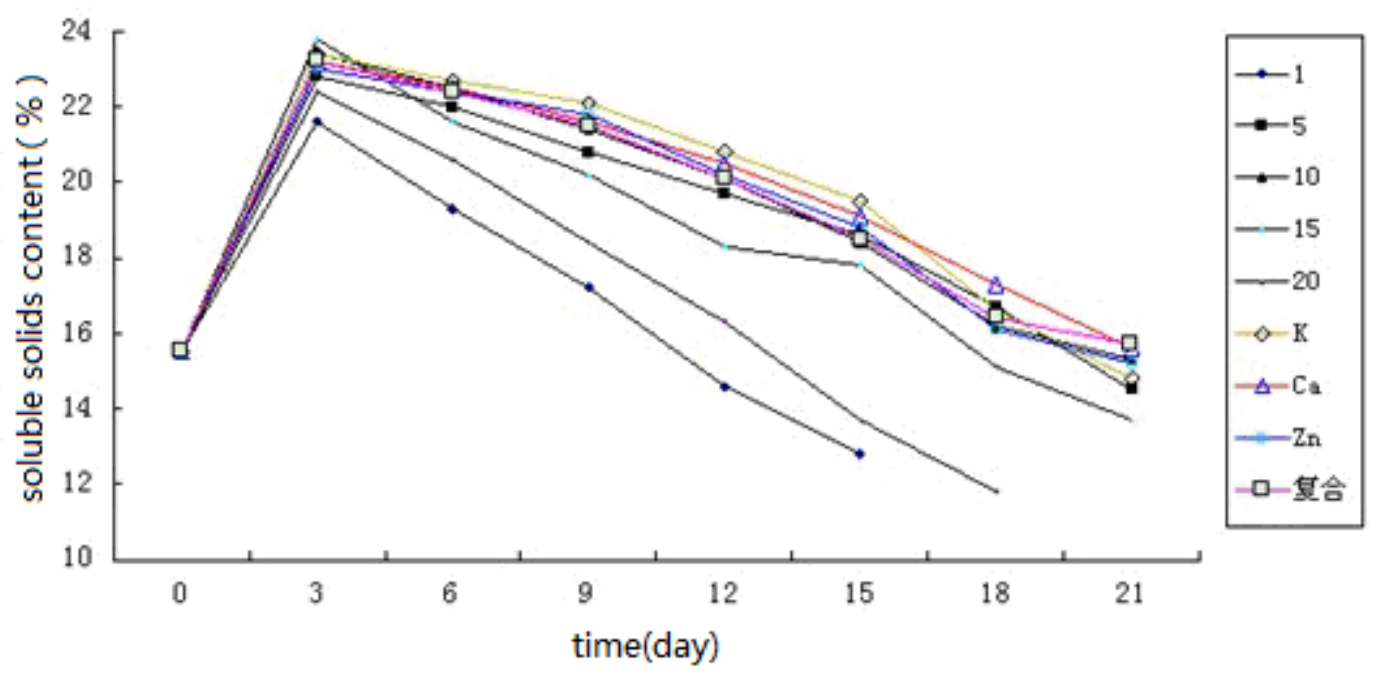

Fig 2 Effect on soluble solids content of cherry

The coating effect of mercapto chitosan and its metal complexes on titratable acid content of cherry

The figure 3 shows the effect of CS coating film on the titratalbe acid content of cherry. The changing trend of titratable acid content is the same as that of soluble solids content. The titratable acid content of experimental groups has some increase at the beginning, which is due to the preservation added a certain concentration of mercapto acid. The figure 3 indicates that the loss of titratable acid is relatively large and basically down to $0.3 \%$.

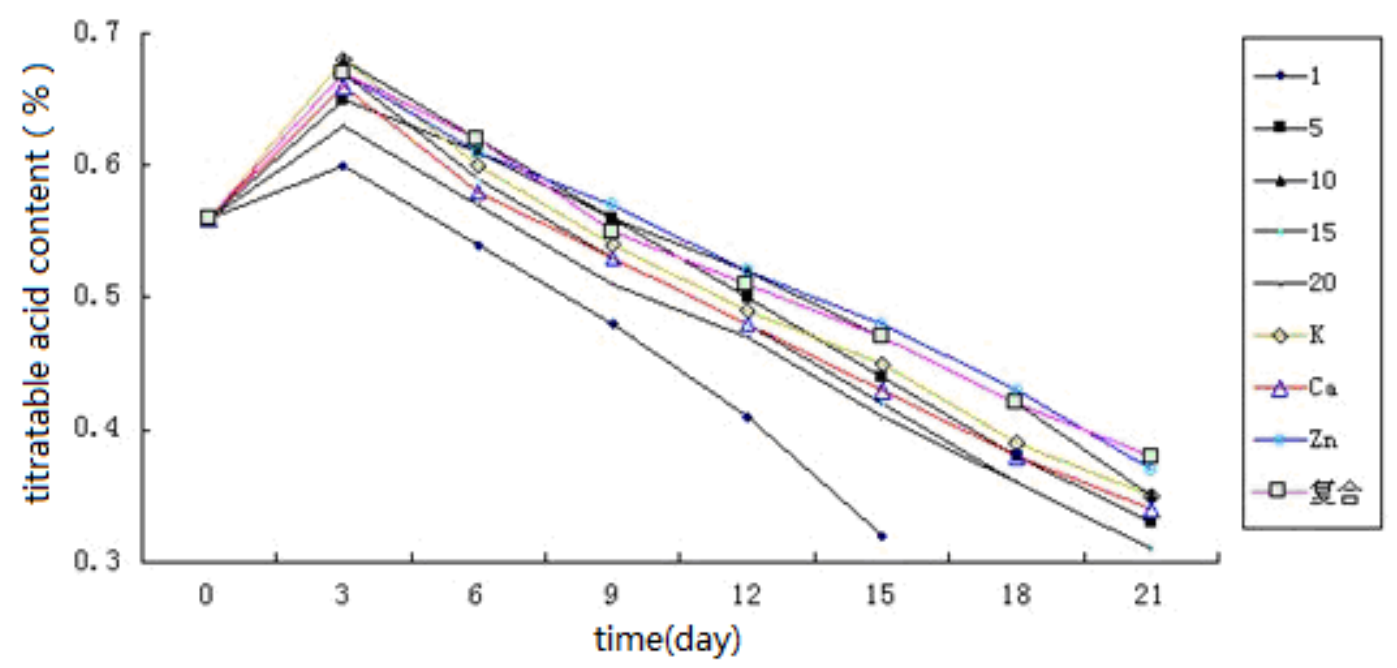

Fig 3 Effect on titratable acid content of cherry

The coating effect of mercapto chitosan and its metal complexes on Vitamin $C$ content of cherry

The figure 4 shows the effect of CS-coated film on ascorbic acid in cherry. Similarly, VC content of the control group declines sharply from the beginning. While, that of the others increases and then decreases. The reason for the phenomenon is that the preservation solution is added ascorbic acid. The experimental data indicates that VC content $(9.56 \mathrm{mg} / 100 \mathrm{~g})$ of coated group is higher than that of control group and the VC content of metal ion complexes group is $10.52 \mathrm{mg} / 100 \mathrm{~g}$. 


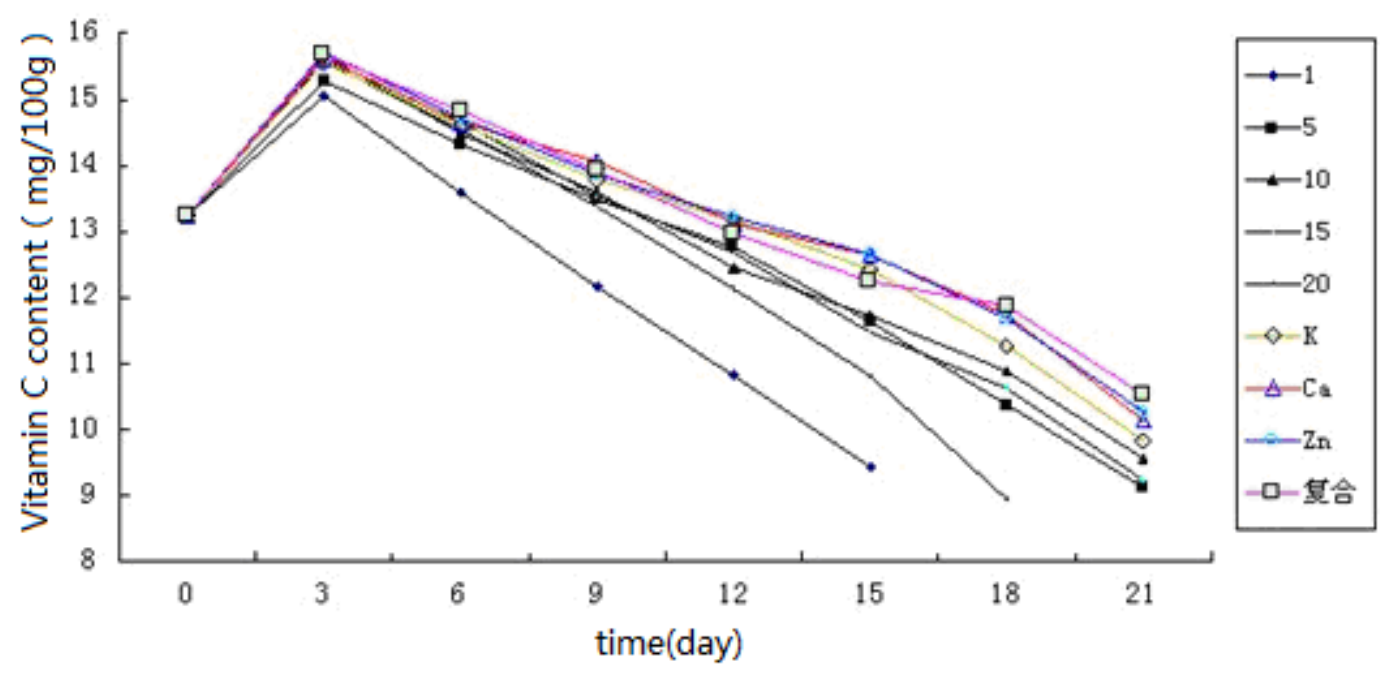

Fig 4 Effect on Vitamin C content of cherry

The coating effect of mercapto chitosan and its metal complexes on respiratory intensity of cherry

The figure 5 shows the effect of CS-coated film on respiratory intensity of cherry. The respiratory rate of cherries which stored up for 3 days is lower than that of previous storage. For all samples, the respiratory rate gradually increases and decreases obviously after storage. The respiratory rate of control group decreases significantly for the second time in 6 days. Previous studies shows that edible coating likes a

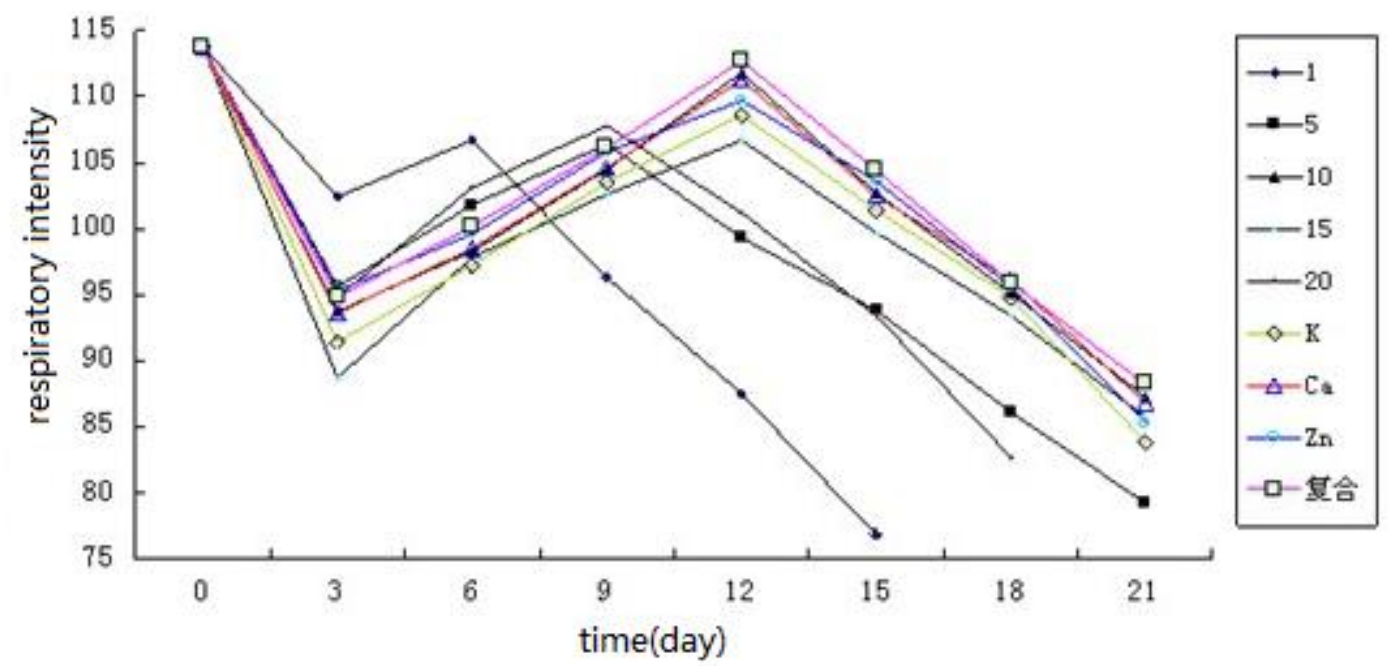

Fig 5 Effect on respiratory intensity of cherry

\section{Conclusion}

The experimental results shows that preservative which prepared by CS can effectively prevent the loss of cherries, soluble solids content, titratable acidity and slow the metabolism of cherry. Besides, the composite preservation that adding certain metal ions (potassium, calcium, zinc)has better effect 
and zinc composite preservation is better. Thereby, the application of chitosan modified materials can effectively maintain the quality and extend the cherry harvest life and the modified chitosan has huge potential in application of coating edible fresh fruit.

\section{References}

[1] Hu Weng-yu. Porperties and Applications on Agriculture of Chitosan[J]. Plant Physiology Journal. 1994, 30(4):294 296.

[2] Jiang Ai-li, Tian Shi-ping.Storing of Cherries[J].Life World, 2002,1: 30.

[3] Yan Jun.Chemistry and Application of Chitsin[J].Chemstry, 1984,(11):26 31 .

[4] Zhang Ai-qunTang Xing-hua,Zhang Ming.Review on the chemical modification of chistosan[J].Chemical Reagent, 2003，25(6): 337 340. 